\title{
On geometry of numbers.
}

\author{
By L. A. Santaló
}

(Received April 1, 1953)

1. Introduction. In a recent paper Tsuji [3] has given some theorems which may be considered as a generalization to Fuchsian groups of the classical theorems of Blichfeldt [1] and Minkowski in the geometry of numbers. In both cases, however, the generalization is restricted to circular domains.

The object of this paper is to carry this generalization further. First, by considering more general groups than the Fuchsian groups; second, by considering a kind of domains more general than the circular domains.

2. A preliminary integral formula. Let $S$ be a space of points in which operates a transitive group of transformations $G$. For a point $P \in S$ and an element $s \in G$ we denote with $s P$ the transform of $P$ by $s$. The elements $s$ of $G$ with $s P_{0}=P_{0}$ for a given point $P_{0}$ of $S$ form a subgroup $G_{0}$ of $G$. Let $G_{1}$ be a simply-transitive subgroup of $G$. Then we have obviously $G_{1} G_{0}=G, G_{1} \frown G_{0}=\{e\}$ (unit group), and we can identify $G_{1}$ with the homogeneous space $G / G_{0}$ or with the space $S$ by assigning $x \in G_{1}$ to $x G_{0} \in G / G_{0}$ or to $x P_{0} \in S . \quad G$ may be then considered as operating on $G_{1}$, as well as on $S$, in the following manner. Let $s, t \in G, x \in G_{1}$, then $s x t$, properly an element of $G$, is identified with the element of $G_{1}$ corresponding to $\operatorname{sxt} G_{0}$ in $G / G_{0}$. We assume now $G_{1}$ to be locally compact and provide $G / G_{0}$ resp. $S$ with the same topology as that of $G_{1}$. We assume further that $G_{1}$ is unimodular, i.e. that the left invariant measure of $G_{1}$ is also right invariant; and that this measure is also invariant with respect to $G$, so that we have $m(H)=m(s H t)$ for a point-set $H$ in $G_{1}$ and any $s, t \in G$. This may be symbolically written as follows $([4,34])$ :

(2.1) $d x=d x^{-1}, \quad d x=d(s x t)$ for any $s, t \in G$,

(2.2) $m(H)=\int_{G_{1}} \varphi(x) d x$,

where $d x$ denotes the element of volume of $G_{1}$ and $\varphi(x)$ the charac- 
teristic function of $H$. By the above identification of $G_{1}$ with $S$, (2.2) gives also the measure of a set $H$ in $S$ if we set $\phi(x)=1$ if $x P_{0} \in H$ and $\varphi(x)=0$ otherwise. Throughout the paper we shall only consider measurable sets.

Let $K_{0}$ be a set of $N$ fixed points $P_{i}(i=1,2,3, \ldots, N)$ of $S$. If we set now $\varphi_{i}(x)=1$ if $x P_{i} \in H$ and $\varphi_{i}(x)=0$ otherwise, according to (2.2) and (2.1) we have

$$
m(H)=\int_{G_{1}} \phi_{i}(x) d x=\int_{G_{1}} \phi_{i}\left(x^{-1}\right) d x
$$

where $\phi_{i}\left(x^{-1}\right)=1$ if $P_{i} \in x H$ and $\varphi_{i}\left(x^{-1}\right)=0$ otherwise.

Let $f\left(P_{i}\right)$ be a function defined on the points $P_{i}$. We have

$$
\begin{aligned}
I & =\int_{G_{1}} \sum_{i} \in x H \\
& =\sum_{1}^{N} f\left(P_{i}\right) d x=\sum_{1}^{N} \int_{G_{1}} f\left(P _ { G _ { 1 } } \phi _ { i } \left(x_{i}\left(x_{i}\right) d x=m(H) \sum_{1}^{N} f\left(P_{i}\right) .\right.\right.
\end{aligned}
$$

In particular, if $f\left(P_{i}\right)=1$ and $\nu\left(K_{0} \cap x H\right)$ denotes the number of points $P_{i}$ which belong to $x H$, we have, [2],

$$
\int_{G_{1}} \nu\left(K_{0} \cap x H\right) d x=N m(H) .
$$

3. An analogue to Blichfeldt's theorem. Let us suppose that there exists a partition of $S$ into fundamental domains $D_{h}(h=$ $0,1,2, \ldots)$ and a discrete subgroup $F$ of $G\left({ }^{*}\right)$ such that:

a) Each $D_{h}$ is the transform of $D_{0}$ by the transformation $x_{h}$ of $F$ $\left(x_{0}=\right.$ unit element of $\left.F\right)$. That is

$$
D_{h}=x_{h} D_{0}, \quad x_{h} \in F .
$$

b) Each $x_{h} \in F$ transforms a fundamental domain $D_{k}$ into a fundamental domain $D_{l}$, with $l \neq k$ if $h \neq 0$.

c) The fundamental domains are measurable and $0<m\left(D_{h}\right)=$ $m\left(D_{0}\right)<\infty$.

Let $K_{0}$ be a set of $N$ points $P_{i}(i=1,2, \ldots, N)$ contained in $D_{0}$ and let $f\left(P_{i}\right)$ be a function defined on the points $P_{i}$ such that $f\left(x_{h} P_{i}\right)=f\left(P_{i}\right)$.

$\left(^{*}\right)$ Note that $F$ is not necessarily a subgroup of $G_{1}$. 
Since $S$ is the sum of the sets $D_{h}$ and the space of the group $G_{1}$ coincides with $S$, the integral $[2.4)$ can be written

$$
I=\int_{G_{1}} \sum_{P_{i} \in x H} f\left(P_{i}\right) d x=\sum_{h} \int_{D_{h}} \sum_{P_{i} \in x H} f\left(P_{i}\right) d x .
$$

By the change of variables $x^{\prime}=x_{h}^{-1} x$, having into account the invariance (2.1), we have

$$
I=\sum_{h} \int_{D_{0}} \sum_{P_{i} \in x_{h} x H} f\left(P_{i}\right) d x=\sum_{h} \int_{D_{0}} \sum_{x_{h}^{-1} P_{i} \mathrm{exH}} f\left(P_{i}\right) d x
$$

and, by (2.4),

$$
\int_{D_{0}} \sum_{h} \sum_{x_{h}^{-1} P_{i} \in x H} f\left(P_{i}\right) d x=m(H) \sum_{1}^{N} f\left(P_{i}\right) .
$$

This means that if we draw on $S$ the lattice formed by all points $x_{h} K_{0}(h=0,1,2, \ldots)$ and for each position $x H$ of $H$ with $x \in D_{0}$, we carry out the addition $\sum f\left(P_{i}\right)$ over the lattice points contained in $x H$ (with the assumption $\left.f\left(x_{h} P_{i}\right)=f\left(P_{i}\right)\right)$, the integral formula (3.4) holds.

Therefore, the mean value of the sum $\sum f\left(P_{i}\right)\left(P_{i} \in x H\right)$ is equal to

$$
\text { m. v. }\left(\sum f\left(P_{i}\right)\right)=\frac{m(H)}{m\left(D_{0}\right)} \sum_{1}^{N} f\left(P_{i}\right)
$$

and we have

THEOREM 1. Let $S, G, G_{1}, F$ be the space and the groups already specified. Given $N$ fixed points $P_{i}$ inside the fundamental domain $D_{0}$, we consider the lattice of all points $x_{h} P_{i}\left(x_{h} \in F\right)$ and a function $f\left(P_{i}\right)$ defined on the points $P_{i}$ such that $f\left(x_{h} P_{i}\right)=f\left(P_{i}\right)$. Then, for every measurable set $H$ of $S$ there are transforms $x^{\prime} H\left(x^{\prime} \in G_{1}\right)$ for which the sum $\sum f\left(P_{i}\right)$ extended over the lattice points contained in $x^{\prime} H$ is not less than the right hand side of (3.5) and transforms $x^{\prime \prime} H\left(x^{\prime \prime} \in G_{1}\right)$ for which that sum is not greater than the right hand side of (3.5).

If $f\left(P_{i}\right)=1$, (3.5) gives that the mean value of the number of lattice points contained in $H$ is $N m(H) / m\left(D_{0}\right)$ and the Theorem gives bounds for the number of lattice points that suitable transforms of $H$ can contain.

If $S$ is the euclidean space, $G$ the group of motions, $G_{1}$ the group 
of translations and $F$ the subgroup of translations which leaves unchanged the lattice of points with integral coordinates, then Theorem 1 coincides with Blichfeldt's theorem.

If $S$ is the unit circle $|z|<1$ of the complex plane, $G$ the group of non-euclidean motions $\left(z^{\prime}=(b z+a) /(\bar{a} z+\bar{b})\right), G_{1}$ the group of noneuclidean translations $\left(z^{\prime}=(z+a) /(1+\bar{a} z),|a|<1\right)$ and $F$ is a Fuchsian group, then Theorem 1 gives the Theorem 5 of Tsuji [3] generalized to domains not necessarily convex.

4. A lemma. With the same notations of $\mathrm{n}^{\circ} 3$, let $H$ be a set of points such that $m(H)>m\left(D_{0}\right)$. Let $D_{i}(i=1,2, \ldots)$ be the fundamental domains which have common point with $H$ and let us consider the set of points $\sum_{i}\left(D_{0} \cap x_{i}^{-1} H\right)$. Since the measure is preserved by $x_{i}$, having into account (3.1), we have

$$
m\left(D_{0} \cap x_{i}^{-1} H\right)=m\left(x_{i}\left(D_{0} \cap x_{i}^{-1} H\right)\right)=m\left(x_{i} D_{0} \cap H\right)=m\left(D_{i} \cap H\right)
$$

and therefore

$$
m\left(\sum_{i}\left(D_{0} \cap x_{i}^{-1} H\right)\right)=\sum_{i} m\left(D_{0} \cap x_{i}^{-1} H\right)=\sum_{i} m\left(D_{i} \cap H\right)=m(H)
$$

As a consequence, since $m(H)>m\left(D_{0}\right)$, the sets $D_{0} \cap x_{h}^{-1} H$ overlap, that is, there is a point $P$ which belong to two sets $D_{0} \cap x_{i}^{-1} H$, say $D_{0} \cap x_{1}^{-1} H, D_{0} \cap x_{2}^{-1} H$. Therefore $P$ belongs to $x_{1}^{-1} H$ and to $x_{2}^{-1} H$ and since $x_{1}^{-1}, x_{2}^{-1}$ belong to $F$, we have the following

LEMMA. If $m(H)>m\left(D_{0}\right)$, then the equivalents of $H$ with respect to $F$ overlap.

For the particular case of Fuchsian groups this lemma coincides with Theorem 1 of Tsuji [3].

5. An analogue to Minkowski's theorem. With the same nomenclature as in $\mathrm{n}^{\circ} 3$, let us now consider the case $N=1$. That is, given a fixed point $P_{0}$ in $D_{0}$, we consider the point lattice $x_{h} P_{0}\left(x_{h} \in F\right)$.

Given a domain $H$ which contains $P_{0}$, we shall say that a domain $H^{*}$ contained in $H$ is an $m$-domain of $H$ (with respect to the group $G$ ) if, for $x \in G, y \in G$, the following two conditions are satisfied:
a) If $x P_{0} \in H^{*}$, then $x^{-1} P_{0} \in H^{*}$.
b) If $x P_{0} \in H^{*}$ and $y P_{0} \in H^{*}$, then $x y P_{0} \in H$. 
Let us assume that $H$ admits an $m$-domain $H^{*}$ such that $m\left(H^{*}\right)>$ $m\left(D_{0}\right)$. Then, by the lemma of $\mathrm{n}^{\circ} 4$, the equivalent sets of $H^{*}$ with respect to $F$ overlap. This means that there exists a point $P=x P_{0}$ which belongs to $H^{*}$ and to a transform, say $x_{1} H^{*}$, of $H^{*}$. That is, we have $x P_{0} \in H^{*}, x P_{0} \in x_{1} H^{*}$, or

$$
x P_{0} \in H^{*}, \quad x_{1}^{-1} x P_{0} \in H^{*} .
$$

Since $H^{*}$ is an $m$-domain of $H$, we have by a)

and by b)

$$
x P_{0} \in H^{*}, \quad\left(x_{1}^{-1} x\right)^{-1} P_{0}=x^{-1} x_{1} P_{0} \in H^{*}
$$

$$
x x^{-1} x_{1} P_{0}=x_{1} P_{0} \in H .
$$

Since $x_{1} P_{0}$ is a lattice point, we have the following

THEOREM 2. If the domain $H$ which contains the point $P_{0}$ possesses an m-domain $H^{*}$ such that $m\left(H^{*}\right)>m\left(D_{0}\right)$, then $H$ contains a lattice point distinct from $P_{0}$.

EXAMPLEs. 1 . Let $S$ be the $\boldsymbol{n}$-dimensional euclidean space, and let now $G=G_{1}$ be the group of translations in it; let $F$ be the subgroup which leaves unchanged the lattice of points with integral coordinates. Let $P_{0}$ be the origin of coordinates and let $H$ be a convex domain with center of symmetry at $P_{0}$. Then it is easy to see that the homothetic domain of $H$ with center $P_{0}$ and ratio $\frac{1}{2}$ is an $m$-domain of $H$. Moreover, if $V$ denotes the volume of $H$, we have $m\left(H^{*}\right)=V / 2^{n}$ and $m\left(D_{0}\right)$ $=1$. Therefore, if $V>2^{n}$, then $H$ contains a lattice point distinct from the origin and we have the classical theorem of Minkowski.

2. Let $S$ be the unit circle $|z|<1$ of the complex plane, $G$ the group of non-euclidean (n. e.) motions $\left(z^{\prime}=(b z+a) /(\bar{a} z+\bar{b})\right)$ and $F$ a Fuchsian group. Let $P_{0}$ be the origin $z=0$. Let $H$ be the disc $|z| \leq$ $\rho<1$ and let $H^{*}$ be the disc $|z| \leq \rho_{\mathrm{J}}$, where $\rho_{0}$ is related to $\rho$ by the equation

$$
\rho=\frac{2 \rho_{0}}{1+\rho_{0}^{2}} .
$$

According to the n.e. metric $d s=2|d z| /\left(1-|z|^{2}\right)$, the n. e. radius of $H^{*}$ is one half of that of $H$. We want to prove that $H^{*}$ is an $m$ domain of $H$, that is, that the conditions a), b) are satisfied. 
a) Let $x$ be the n. e. motion $z^{\prime}=(b z+a) /(\bar{a} z+\bar{b})$. Since $P_{0}$ is the origin $z=0$, the point $x P_{0}$ is the point $z^{\prime}=a / \bar{b}$. The point $x^{-1} P_{0}$ is $z^{\prime \prime}=-a / b$ and therefore if we assume $\left|z^{\prime}\right|=|a / b| \leq \rho_{v}$, we have $|z|=|a / b|=\left|z^{\prime \prime}\right| \leq \rho_{0}$.

b) Let $r$ be the n.e. length of $\rho$ and $\sigma$ the n. e. length of the segment which unites $P_{0}(z=0)$ with the point $y P_{0}$; if we assume $y P_{0} \in H^{*}$, then we have $\sigma \leq r / 2$. Since the n. e. motions preserve the n. e. lengths, the n. e. length of the segment (arc of geodesic) which unites the points $x P_{0}$ and $x y P_{0}$ is equal to $\sigma$. Therefore, from $x P_{0} \in H^{*}$ and $\sigma \leq r / 2$ we deduce $x y P_{0} \in H$.

Consequently $H^{*}$ is an $m$-domain of $H$ and Theorem 2 can be applied. Having into account that, in this particular case, we have $m(H)=4 m\left(H^{*}\right)+\pi^{-1} m^{2}\left(H^{*}\right)$, if $m(H)>4 m\left(D_{0}\right)+\pi^{-1} m^{2}\left(D_{0}\right)$, then $H$ contains a lattice point distinct from $z=0$, and we have the theorem 2 of Tsuji [3],

\section{Faculty of Sciences, University Eva Peron Argentina.}

\section{References}

[1] H.F. Blichfeldt, A new principle in the geometry of numbers with some applications. Trans. American Math. Soc., 15 (1914), 227-235.

[2] L. A. Santalô, Integral Geometry in general spaces. Proc. of the International Congress of Math. 1950, vol. 1, 483-489.

[3] M. Tsuji, Theorems in the geometry of numbers for Fuchsian groups. Journal of the Math. Soc. of Japan, 4 (1952), 189-193.

[4] A. Weil, L'integration dans les groupes topologiques et ses applications. Actualités Scientifiques et Industrielles, Hermann, Paris, 1940, n869. 